\title{
Tracking in the trigger: from the CDF experience to CMS upgrade
}

\author{
Fabrizio Palla ${ }^{1}$ \\ INFN Pisa \\ Largo B. Pontecorvo 3, 56127 Pisa, Italy \\ E-mail:Fabrizio.Palla@cern.ch
}

\section{Giuliano Parrini}

University and INFN Florence

Via G. Sansone 1, 50019, Sesto Fiorentino (Firenze), Italy

E-mail:parriniefi.infn. it

\begin{abstract}
Precise tracking information in the online selection of interesting physics events is extremely beneficial at hadron colliders. The CDF experiment at the Tevatron, has shown for the first time the impact of the tracking in triggers, allowing to achieve unprecedented precision in B-physics measurements. The CMS experiment at LHC will largely make use of tracking information at high level trigger, after the Level-1 acceptance. The increased luminosity of the Super-LHC collider will impose to CMS a drastic revision of the Level-1 trigger strategy, incorporating the tracker information at the first stage of the selection. After a review of the CDF and CMS approaches we will discuss several possible Level-1 tracker based concepts for the upgraded CMS detector at Super-LHC. One approach is based on associative memories, which has already been demonstrated in CDF. It makes use of binary readout in the front end electronics, followed by transfer of the full granularity data off detector using optical links to dedicated processors which reconstruct tracks.
\end{abstract}

The 16th International Workshop on Vertex detectors

Lake Placid, NY, USA

23-28 September, 2007

1 Speaker 


\section{Introduction}

Experience at high luminosity hadron collider experiments shows that controlling trigger rates can be extremely challenging as the luminosity increases, physics goals change in response to new discoveries, and the detector ages. It is thus essential that the trigger system be flexible and robust, and have redundancy and significant operating margin.

Information from the inner tracking detectors is highly beneficial in achieving these goals. This has certainly been the case in the CDF experiment where the Silicon Vertex Trigger [1,2] (SVT) has significantly extended the experiment's physics capability.

Even more challenging will be the trigger for the foreseen upgrade of LHC, the so-called SuperLHC (SLHC) Luminosity increase of a factor 10 with respect to the "standard" LHC run [3]. The increased luminosity will be reached at the expenses of producing about 400 collisions per bunch crossings, every $50 \mathrm{~ns}$. This will demand unprecedented trigger capabilities in order to drastically reduce the rate of non interesting events, without compromising the luminosity upgrade. Raising the thresholds of the muon and calorimeters triggers at Level-1 will not be sufficient, at least for CMS. In the current trigger architecture, the inclusion of the Tracking information at High Level Trigger improves the rejection by three orders of magnitude. It is therefore natural to design the new Level-1 Trigger based on Tracker detector, that needs to be rebuilt anyway for operating at SLHC.

This paper will present how the tracking trigger information has demonstrated to play a key role in the physics of CDF and some ideas on current studies for CMS at SLHC. Section 2 will illustrate the effectiveness of a tracking trigger operated at Level-2 in CDF, the pioneer of tracking triggers at hadron colliders. Section 3 will illustrate the issues of a Level-1 trigger with Tracker for CMS at SLHC. Finally in Section 4 we will discuss a proposal for a Tracking trigger at Level-1 for CMS at SLHC using associative memories approach.

\section{Trigger with tracks in CDF}

SVT performs online reconstruction of tracks crossing the silicon detectors (SVX) and the central drift chamber (COT), with sufficient accuracy to allow recognizing tracks from b-quark decays thanks to their larger impact parameters measured $(>100 \mu \mathrm{m})$ with respect to the beam axis. SVT was designed and built for Bphysics applications for the Tevatron intermediate luminosity RUN IIA $\left(\sim 10^{32} \mathrm{~cm}^{-2} \mathrm{sec}^{-}\right.$ ${ }^{1}$ ) and had an extremely significant impact on the CDF physics program: it promoted the general-purpose CDF experiment to one of the major world players in the field of Bphysics. Among many B-physics results, SVT has been essential to collect the data used for the first measurement of the mixing frequency of the $B_{s}$ system. This would have been completely out of the CDF reach without the large samples of hadronic B decays triggered by SVT. 


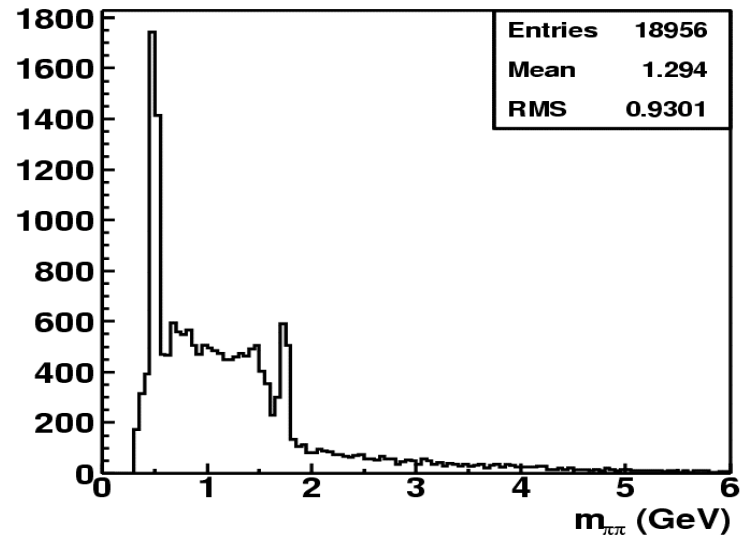

Figure 1. Two pion invariant mass plot in CDF

All tracks with transverse momentum above $2 \mathrm{GeV} / \mathrm{c}$ are reconstructed by the SVT with the maximum spatial resolution allowed by the silicon vertex detector. Figure 1 shows the two pion track invariant mass distribution as reconstructed online with SVT. The $\mathrm{D}^{0}$ peak is clearly visible and is used to monitor the SVT efficiency run by run. The $5 \mathrm{GeV} / \mathrm{c}^{2}$ mass region shows a very low level of background.

Historically, B-physics events have been selected at hadron colliders by triggers based on lepton identification. Trigger selections based on the reconstruction of secondary decay vertices increase the b-quark identification efficiency and allow collecting also the hadronic decay modes, otherwise inaccessible to lepton triggers. The capability to use hadronic b-quark decays determined the difference in performances between the CDF and D0 results for example in the $\mathrm{B}_{\mathrm{s}}$ mixing measurement (see Fig. 2).
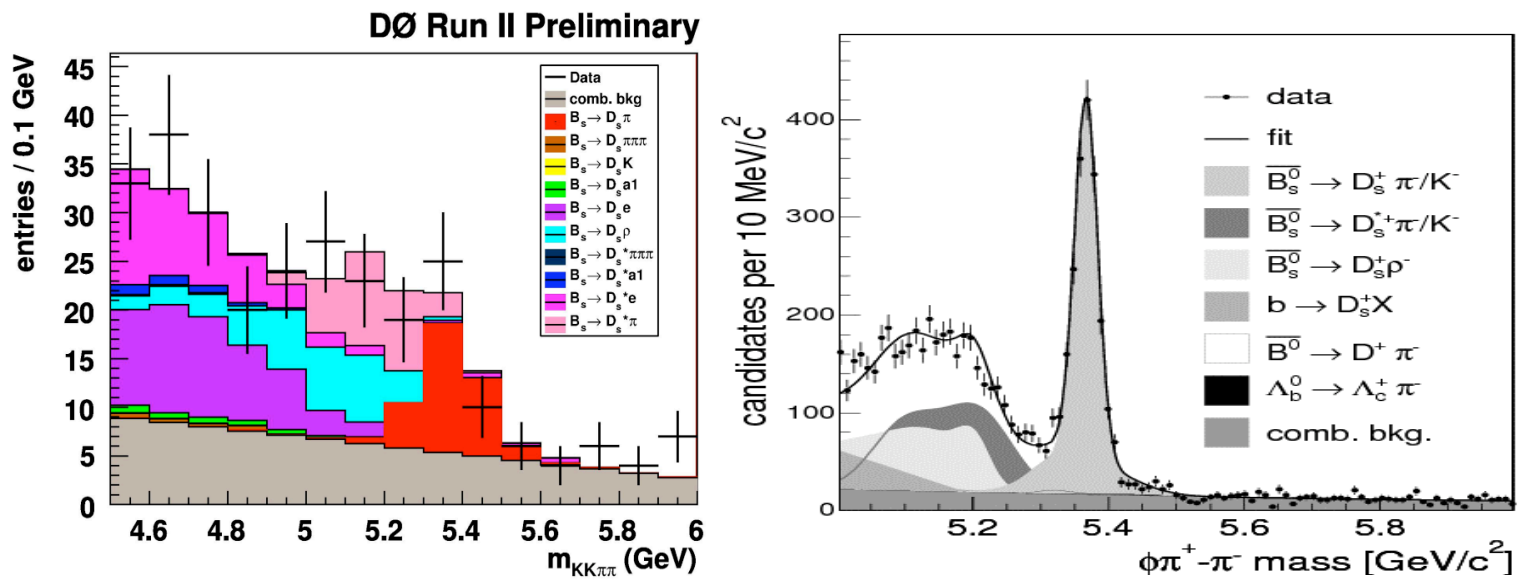

Figure 2. Reconstructed invariant mass plots of $B_{s}$ mass candidates decaying into a $D_{s}$ and a pion, together with the contribution from other final states. The sharp peak from CDF (right) is compared to the less pure sample found by D0 (left). 


\subsection{The SVT architecture and the Associative Memory at CDF}

The eXtremely Fast Tracker (XFT) finds tracks with transverse momentum above $1.5 \mathrm{GeV} / \mathrm{c}$ in two dimensions ( $\mathrm{r}-\phi)$ at Level-1 (LVL1) in the Central Outer drift Chamber (COT) with $96 \%$ efficiency, transverse momentum resolution in $1 / \mathrm{p}_{\mathrm{T}}$ of $1.7 \%$ and angular resolution of $5 \mathrm{mrad}$ in phi. In addition to being used in the LVL1 trigger decision, the XFT tracks are distributed to the SVT for subsequent use at Level-2. The SVT links the hits found on the five layers of the silicon vertex detector to the XFT tracks. The complex pattern recognition algorithm is subdivided in two simpler sequential steps of increasing resolution. The first step associates the silicon hits and XFT tracks with low spatial resolution track candidates, called roads. A dedicated device called Associative Memory (AM) [4] performs this first function. In the second step, the real tracks are searched within the roads and fitted to determine their parameters (transverse impact parameter resolution $35 \mu \mathrm{m}, \sigma(1 / \mathrm{Pt})=0.3 \%$ and $\sigma(\varphi)=1$ mrad). Tracks with a large impact parameter (distance larger than $100 \mu \mathrm{m}$ from the beam line) are finally selected to tag the presence of a secondary vertex.

The basic Associative Memory chip has been made with VLSI full-custom technology [5] and it is specific of the CDF 5-layer silicon detector. The AM takes full advantage of the parallel nature of the problem by simultaneously comparing the tracker data to all the stored patterns. This approach reduces the typical exponential complexity of pattern recognition with CPUs to a simple linear dependence only on the input data size. The problem is solved within the time needed to load the data into the device. The SVT includes important pre/post processing functions, complementary to the intensive pattern-recognition. Pre-processing corresponds to cluster finding in the silicon data and a smart database for immediate retrieval of full resolution information. Post-processing includes the track fitting (TF) and duplicate-track cleanup (ghost busting). The most relevant function is the track fitting (TF), which refines the candidate tracks in order to determine the track parameters with the full detector resolution. The TF makes use of methods based on local linear approximations and learn-from-data techniques for online misalignment corrections. The SVT experience shows that the approximations introduced to maximize the speed often do not significantly affect the fit performance.

\section{CMS Tracker Trigger for Super LHC}

The proposed LHC luminosity upgrade [3] - also called Super LHC (SLHC) will allow the increase of the luminosity to $10^{35} \mathrm{~cm}^{-2} \mathrm{~s}^{-1}$ with an increase up to about 400 proton-proton interactions per bunch crossing for a collision frequency of $20 \mathrm{MHz}$. This would correspond to about 10 billions of collision events per second, to be compared to the rare physics phenomena which will occur at about few Hertz or lower. Each proton bunch crossing will generate much more than the present $1 \mathrm{MB}$ of data, and the expected data transfer to disk will not exceed the rate of about $100 \mathrm{~Hz}$. Hence, the increased luminosity will also require a drastic revision of the existing trigger strategies. As an example, Fig. 3 shows the rate of the single muon trigger as a function of the muon $\mathrm{p}_{\mathrm{T}}$ threshold [14]. The performance at First Level Trigger suffers from poor transverse 
momentum resolution at high $\mathrm{p}_{\mathrm{T}}$. The use of the Tracker information at High Level Trigger (labelled as Level 3 in Fig. 3) allows a large change in the slope of the rate.

Due to the shape of the underlying background distributions, the required Level1 rate reduction for SLHC cannot be achieved by increasing the Level-1 thresholds, even if one is willing to pay the price of reducing the rate of useful events. Gain in background reduction can only come from improving the Level-1 algorithms for selection of physics signals. Furthermore, at the SLHC the Level-1 efficiency and purity to select Higgs and other exotic signals will degrade due to the increase of the minimum bias events by at least a factor of 5. Given that the current Level-1 design already uses information from all detectors but the silicon tracker, any improvements in background reduction efficiency and purity can only come by including tracking information at the first level trigger. Hence, the need for a First Level Tracking Trigger.

\subsection{Tracker Trigger issues for CMS at SLHC}

Any tracking trigger will have to cope with the huge amount of data that will be produced at SLHC. Simulations based on Pythia tune A [8] show that the expected number of charged tracks per bunch crossing (50 ns) at SLHC will be of the order of about 12,000 with about 400 primary vertices in a luminous region of about $20 \mathrm{~cm}$, in the region where the current CMS Tracker is located $(|\eta|<2.5)$. The uncertainty on these numbers are of the order of tens percent, hence giving potential increase in the particles that is difficult to quantify. The fluence, from both charged and neutral particle hits, in the detectors is such that even at $30 \mathrm{~cm}$ the expected hit rate is about $50 \mathrm{MHz} \mathrm{cm}$, while at 1 meter is of the order of $3 \mathrm{MHz} \mathrm{cm}$.

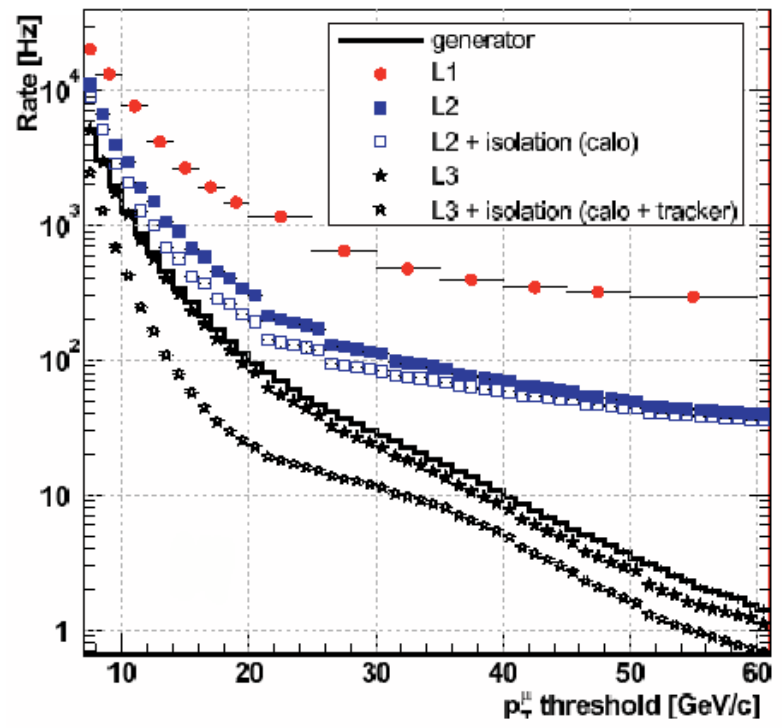

Figure 3. The Level-1 Trigger and High Level Trigger (HLT)single-muon rates as a function of the $p_{T}$ threshold at LHC. The rates are shown separately for Level-1 and HLT (Level-2, and Level-3), with and without isolation applied at Levels 2 and 3. The rate generated in the simulation is also shown. 
Assuming a binary readout of the trigger information and 18 bits the coordinates

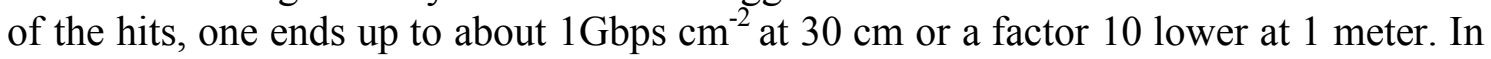
addition, if the time stamp is needed then the word increases by 12 bits. Hence, the real challenge is to make such a large amount of data available in time to a tracking processor.

From the design point of view, coping with the large data rate could be accomplished in several ways: fulfilling the required high speed transmission of all the data to the trigger processors, work at larger radii and reduce the information to be transmitted by means of some local data reduction. In addition, coarser granularity or selective readouts could also be possible, but the evaluation of their benefits is still under study for SLHC. In the following, we will concentrate only on the first three ways.

The data rate transmission speed needed to transfer the data outside of the detector volume, to dedicated Trigger hardware, as proposed in [9] is challenging. Current prejudices indicate that in order to guarantee sufficient operating margins the required speed of the devices should be about $10 \mathrm{Gbps}$ for layers, even at large radii, and would demand quite substantial R\&D and costs. Commercially available technologies could be potentially attractive for their large speed (several tens of Gbps) but are not proven to be radiation hard and the power consumption needs to be investigated. On the other hand, current R\&D has focused on multipurpose devices, but proved to be radiation hard and limited power consumption [10], with a useful data bandwidth of about $2.5 \mathrm{Gbps}$, based on a $130 \mathrm{~nm}$ technology: if developed in 90 or $65 \mathrm{~nm}$ technology, the chip could possibly match the requirement [11], but the power budget implication is unknown. Despite the attractiveness of the proposal, one is forced realistically to explore alternative paths that require data reductions at the level of the data to be sent to the trigger processors. It should be noted however that having fast data transfer will ease the complexity of the system.

Using the information of the detectors at sufficiently large radii would certainly help, not only in terms of data rate but also for good momentum measurement, since the momentum resolution scales inversely quadratic with the lever arm. Increasing the lever arm would inevitably reduce the impact parameter resolution. However, as shown in Figure 4, the current CMS simulation indicates that reconstructing the tracks with only the information of the strip detectors (that have their first measurement point at about $25 \mathrm{~cm}$ from the beam line) gives a transverse impact parameter resolution of the order of a few hundred micron and about a $\mathrm{mm}$ in the $\mathrm{r}-\mathrm{z}$ plane. This situation could only be better at SLHC, because of the finer pitch needed to cope with the increased occupancy. One could restrict the $\mathrm{z}$ origin of the tracks (and therefore of the possibly matched muons, electrons or jets associated to them) in a region of only a few vertices out of the 400 in about $20 \mathrm{~cm}$ S-LHC luminous region. Similarly, one could reject muons from pion and Kaon decays by using the transverse impact parameter of a track.

Hence, while the main usage of a Tracking Trigger is intended for reconstruction of particles exceeding a given transverse momentum threshold, and to match it with muons or electrons, the usage of the best track resolution is highly beneficial. 
The other approach to reduce the formidable data rate is to have some local data reduction on the module. This could be achieved by using only the hits coming from the tracks with sufficient large momentum, as the rare events of interest have particles with $\mathrm{p}_{\mathrm{T}}$ above several GeV/c and only about $5 \%$ of the minimum bias particles have $\mathrm{p}_{\mathrm{T}}>2$ $\mathrm{GeV} / \mathrm{c}$ [8]. Two different approaches are currently pursued to achieve such data reduction, both exploiting the fact that tracks with low $\mathrm{p}_{\mathrm{T}}$ enter the sensitive silicon detectors with larger angles with respect to the measurement plane, compared to higher momentum tracks that are almost perpendicular to it. In the following we will concentrate only in one of the two methods [12], the other one being illustrated in another talk at this conference [13].
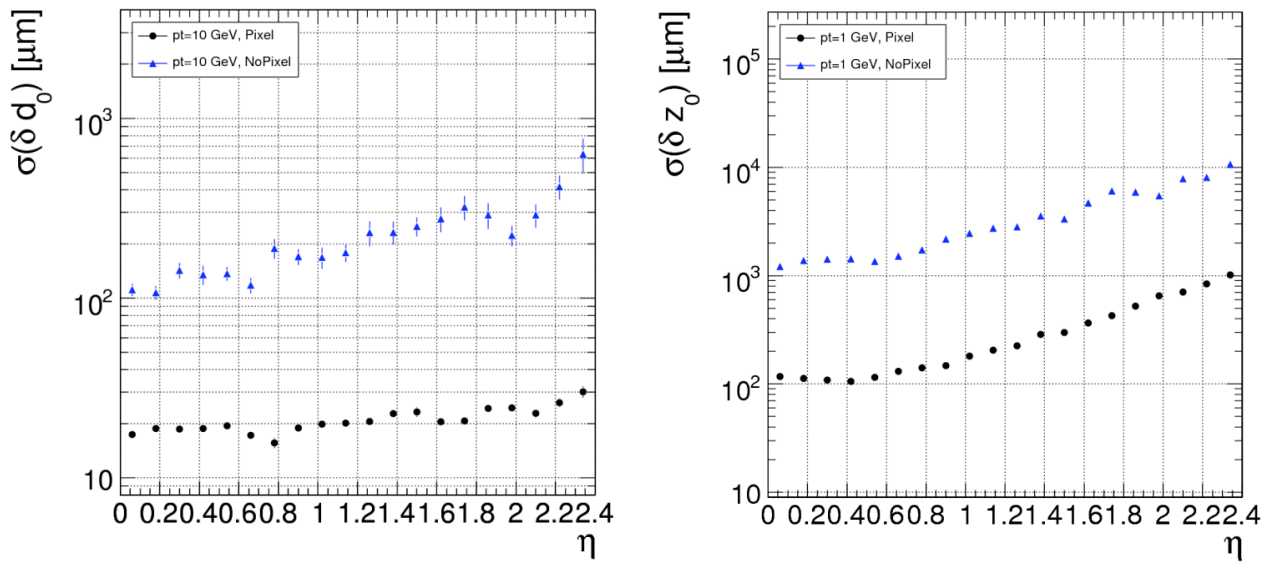

Figure 4. Transverse impact parameter (left) and longitudinal impact parameter (right) for single muon tracks in the CMS Tracker as a function of the pseudorapidity $\eta$. Black dots show the full tracker reconstruction with pixels, blue triangles without.

\subsection{Discriminating low momentum tracks using track cluster width}

In the current proposal, for sake of simplicity, only the barrel region is considered, covering the pseudorapidity region of $|\eta|<1.5$. All problems linked to the mechanics, cooling or silicon sensor technologies, go beyond the scope of this contribution. It is sufficient to state that in this region, both the radiation hardness as well as services will not pose a big problem as for the layers below $15 \mathrm{~cm}$ radius. With a suitable extension of the sensor concept it can be used with thinner sensors as well as in the end caps of the tracker, but this is not discussed here.

In the barrel the sensors are arranged in concentric cylindrical layers of finite length, which are both coaxial with the beams axis and magnetic field. For illustrative purposes we consider that the sensors will be equipped with short strips or long-pixels (order of a few $\mathrm{mm}$ to few $\mathrm{cm}$ ) and fine pitch readout (from 50 to 100 micron).

The principle of the method is sketched in Fig. 5 and relies upon the fact that low $\mathrm{p}_{\mathrm{T}}$ tracks cross the sensors with inclined angles in the r-phi projection plane. The projected "track width" (TW) on the surface where the strips are located is an analytical function of the track $\mathrm{p}_{\mathrm{T}}$, the radial distance $R$ of the sensor and its thickness $t$ : 


$$
T W_{0} \sim \frac{0.15 \times B \times R \times t}{p_{T}} .
$$

where $\mathrm{B}$ is the magnetic field (4T in CMS).

read out electrodes

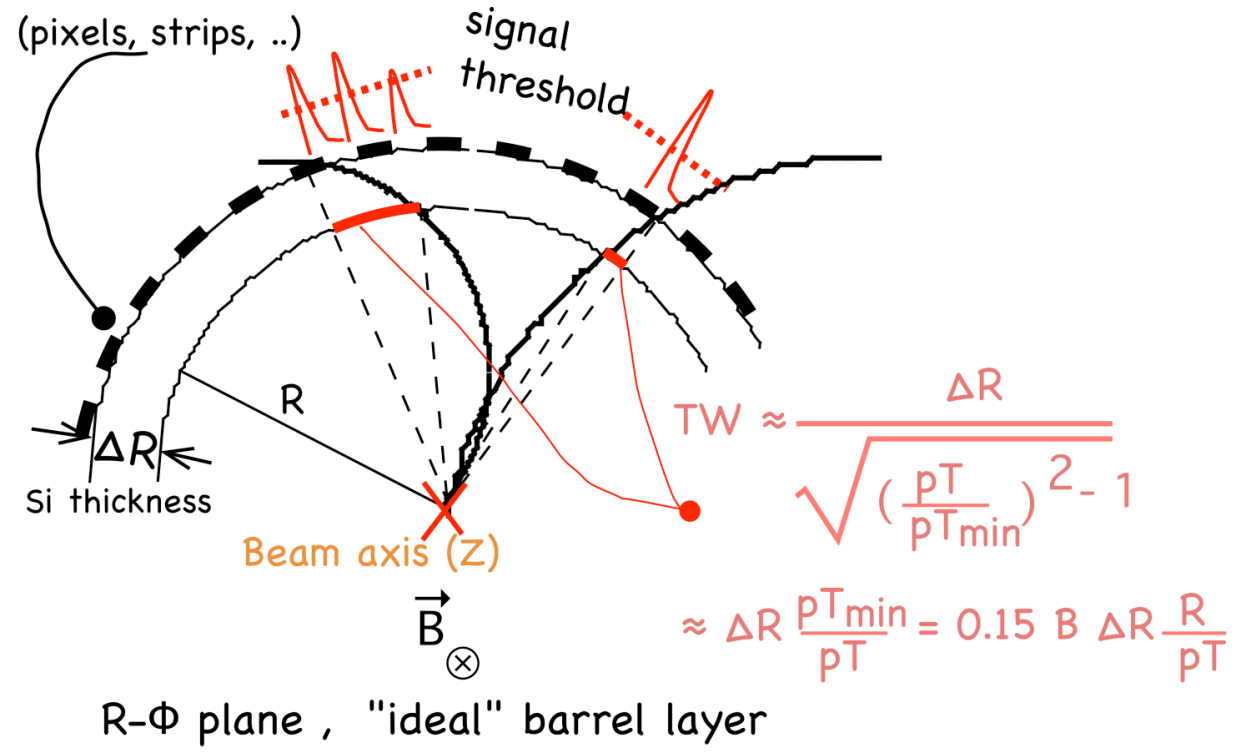

Figure 5. The basic of the track width method [12].

Not surprisingly, the track width is proportional to the radial distance of the sensor and inversely proportional to the track momentum. For a "standard" sensors thickness of $300 \mu \mathrm{m}$ this corresponds to a track width of 90 microns for a $1 \mathrm{GeV} / \mathrm{c}$ track at $50 \mathrm{~cm}$. Tracks with momentum exceeding $10 \mathrm{GeV} / \mathrm{c}$ would instead have a track width of less than $9 \mu \mathrm{m}$.

Actually, silicon sensors are flat and barrel layers are cylindrical assemblies of Silicon tiles which can be tilted around the beam axis by an angle either to partially compensate for the Lorenz angle or for a residual misalignment of the mechanics. This makes the behaviour of the track width more complex than in the case of Fig. 5. For particles hitting the sensor near its centre the TW can be approximated with a linear function of the coordinate of the impact point, as shown in Fig. 6. It is clear that the net separation between the $3 \mathrm{GeV} / \mathrm{c}$ and the $1 \mathrm{GeV} / \mathrm{c}$ track lines allows to discriminate low $\mathrm{p}_{\mathrm{T}}$ particles from those at higher $\mathrm{p}_{\mathrm{T}}$ and to considerably reduce the amount of data needed for the trigger decision.

The implementation of this data reduction in a real detector is achieved by designing sensors with suitable pitch equipped with an ad-hoc logic in the front end readout, capable to perform some elementary "cluster analysis". For instance for a strip pitch of $60 \mu \mathrm{m}$ the discrimination of low $\mathrm{p}_{\mathrm{T}}$ tracks could be achieved by sending to the trigger processor only those hits with less than 2 strips in the cluster (on-strip cluster). 


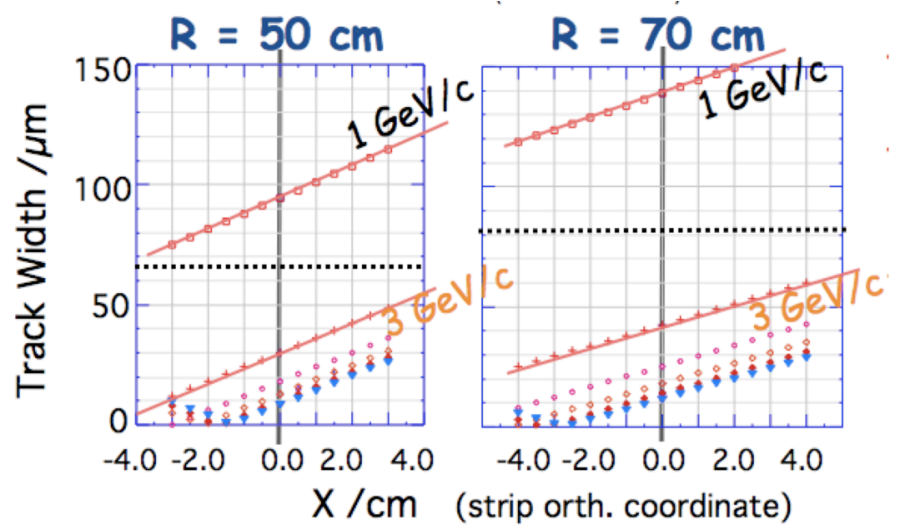

Figure 6. Track width as a function of the impact coordinate $X$ on the detector, for particles of the same charge passing through $300 \mu \mathrm{m}$ thick sensor. Dashed lines indicate the discrimination values between low and high $\mathrm{p}_{\mathrm{T}}$.

This implies the development of an ad-hoc electronics as sketched in Fig. 7, where the comparator is controlled by the enable input and needs a pedestal subtraction to be done. The electronic should be able to perform pedestal subtraction. Both the comparator and the pedestal circuitry receive the output of the amplifier. The result of the comparison is fed to the combinatorial circuitry on the detector - not shown in the Figure - that recognises the "one-strip cluster" and allows the position of the hit to be sent to the trigger logic. The same combinatorial circuitry could handle also the selection based on "two-strips clusters".

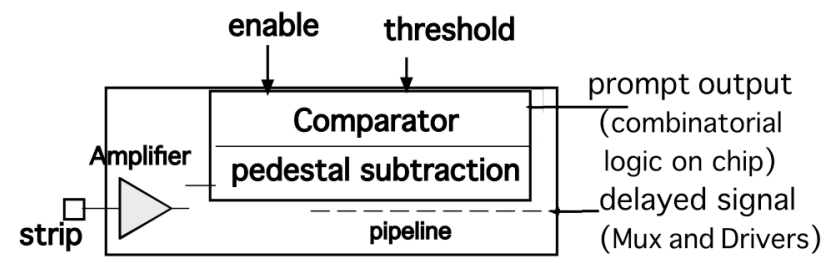

Figure 7. Functional block diagram of the channel electronics.

To verify the feasibility of the discrimination of medium-high $\mathrm{p}_{\mathrm{T}}$ among all other low $\mathrm{p}_{\mathrm{T}}$, a fast simulation of minimum bias events has been performed. The particles are bent by a $\mathrm{B}=4 \mathrm{~T}$ field and can reach the silicon flat sensors placed at several distances, in the pseudorapidity interval $|\eta|<1.5$ The measurements of the track widths are performed comparing the signals of the strips with a constant threshold. The number of contiguous strips exceeding the threshold (cluster) gives the TW measure. This algorithm is a simplified version of the one used for the cluster finding in strip sensors where the search is done using two thresholds at least, one for the "seed" strip, one for the next ones. The threshold is set to 4 times the average noise.

The $\mathrm{p}_{\mathrm{T}}$ spectra of a minimum bias particle sample when passing through sensors at different distances from the beam are shown in Fig. 6. Selecting particles with TW= 1 strip ("one-strip cluster") heavily suppress tracks with $\mathrm{p}_{\mathrm{T}}<2 \mathrm{GeV} / \mathrm{c}$. The total rejection is good, of the order of $90 \%$ or better, and it shows a good stability with respect to the pitch values. 
cluster width $>1$ strip rejection, signal threshold at $4 \times$ onoise

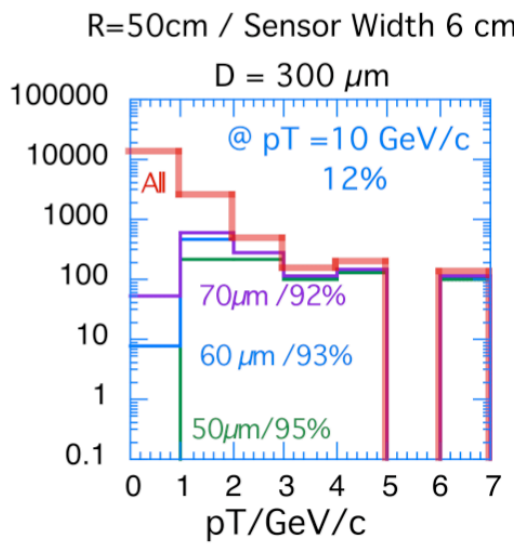

$\mathrm{R}=70 \mathrm{~cm} /$ Sensor Width $9 \mathrm{~cm}$

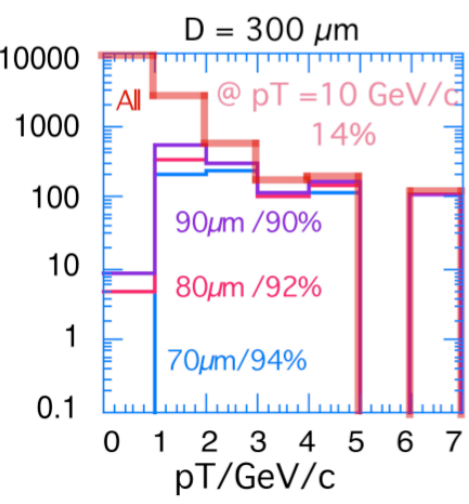

Figure 6. $\mathrm{p}_{\mathrm{T}}$ spectra (thick red lines) of a minimum bias particles sample in a 4T magnetic field from sensor thickness of $300 \mu \mathrm{m}$ at two different distances $(\mathrm{R})$ and sensor widths. Thin lines show the spectra of 1 strip only clusters $(4 \sigma$ threshold) for three different pitches. The "rejection" refer to particles with $p_{\mathrm{T}}=10 \mathrm{GeV} / \mathrm{c}[12]$.

In order to quantify the gain in terms of reduction in the number of hits, one should note that a track with low momentum is fairly large, giving rise to large number of hits per track. For instance, for at $50 \mathrm{~cm}$ radius the average track width in minimum bias events is about $0.3 \mathrm{~mm}$ with a rms of $0.7 \mathrm{~mm}$, and it increases to an average of 0.5 $\mathrm{mm}$ at $70 \mathrm{~cm}$ with a dispersion of about $1.5 \mathrm{~mm}$. Typically, for a track reduction of $90 \%$ the gain in the number of hits is about 5 or more when a r-phi pitch of $60 \mu \mathrm{m}$ is chosen.

More studies are required to prove the effectiveness of such a simple method in real data. In particular, the influence of charge diffusion, capacitive couplings also associated with radiation induced effects. Extensive R\&D is planned in future.

\section{An Associative Memory based Level-1 Trigger for CMS at SLHC}

The working idea for a tracking trigger is to provide tracks above a given momentum threshold that could be matched with first level trigger muons or electrons in the Global Trigger.

As stated in the previous section, the real challenge is to make such a large amount of data available in time to a tracking processor and some ideas were discussed. In addition, data will be sent to the trigger exploiting a parallel readout of the chosen detector layers. In order to sustain very high event rates, it is necessary to organize the tracking processor as a set of independent engines, each working on a different section of the silicon tracker.

The conceptual scheme for the implementation of the trigger is sketched in Fig. 7. The detector will be divided into many azimuth sectors (order of 100). Each sensor in a sector will send the information to a specific engine which is located outside the detector and will combine the information from the layers to identify the track. Any hit 
in each layer of the sector will be identified by a word defining both the position in the detector and the time-stamp (the bunch crossing number).

The engine is an associative memory chip (AM chip) where a set of all possible tracks (exceeding a given $\mathrm{p}_{\mathrm{T}}$ cut and originating from a certain luminosity region compatible with the beam spot) has been previously loaded. Tracks are pre-calculated taking into account multiple scattering; these are stored in the pattern bank. Each stored hit pattern is provided with the necessary logic to compare itself with the event. The size of the bank pattern depends on the number of detectors in each Trigger sector and their granularity.

Each engine working on a particular $\phi$ sector will receive hits from each layer in parallel and will produce track candidates. The reduced information coming from the sensors in each sector will need a special multiplexer ASIC, inside the detector, to distribute them off detector, where the trigger engines will be located.

The hit information from overlap regions of neighbouring sectors could be sent to more than one engine to avoid efficiency losses in the boundaries between sectors.

To participate in the Level-1 Trigger decision, the sub-detectors must provide relevant information concerning objects of interest to the global trigger within the Level-1 latency time. The track processor latency is dominated by time needed to load hits inside the AM bank. The current AM chip runs at $50 \mathrm{MHz}$ with 6 parallel buses of 18 bits encoding each. It is envisaged that the future evolutions of the AM chip will run at 100-200 MHz. Even if the new AM chip will be substantially faster than the old one, it is probably too slow to be able to load high-occupancy events within the level-1 latency in the SLHC conditions.

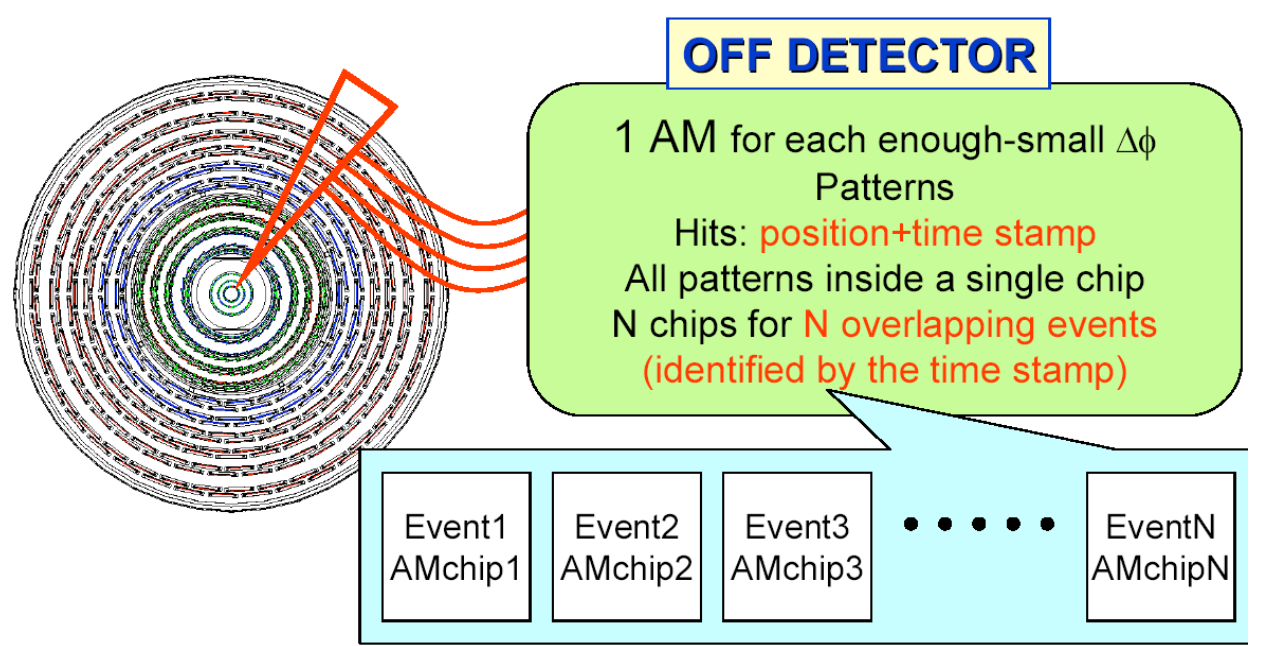

Figure 7. Conceptual scheme for a Tracker Trigger using Associative Memory like approach.

For this reason the flux coming from a layer $\phi$ slice is sent to a switch that distributes the incoming hits into different sets of storage units as shown conceptually in Fig. 8, depending on the event number. The registers are enough to store up to $\mathrm{N}$ events at the same time. In this way a number $\mathrm{N}$ of AM chips will work in parallel on different 
events, together being able to sustain the input rate, $\mathrm{N}$ times larger the input bandwidth of a single chip. Each single $\phi$ slice layer is readout in parallel by many different fibres to sustain the high hit rate. Each fibre is received on a dedicated FIFO, from where hits are moved to registers classified by event number, to be finally mutiplexed on a single bus for the AM chip. The switch logic, even if conceptually simple, is the most demanding part of the system in term of working frequency; FPGA technology can work up to $500 \mathrm{MHz}$ and is the best candidate for possible R\&D.

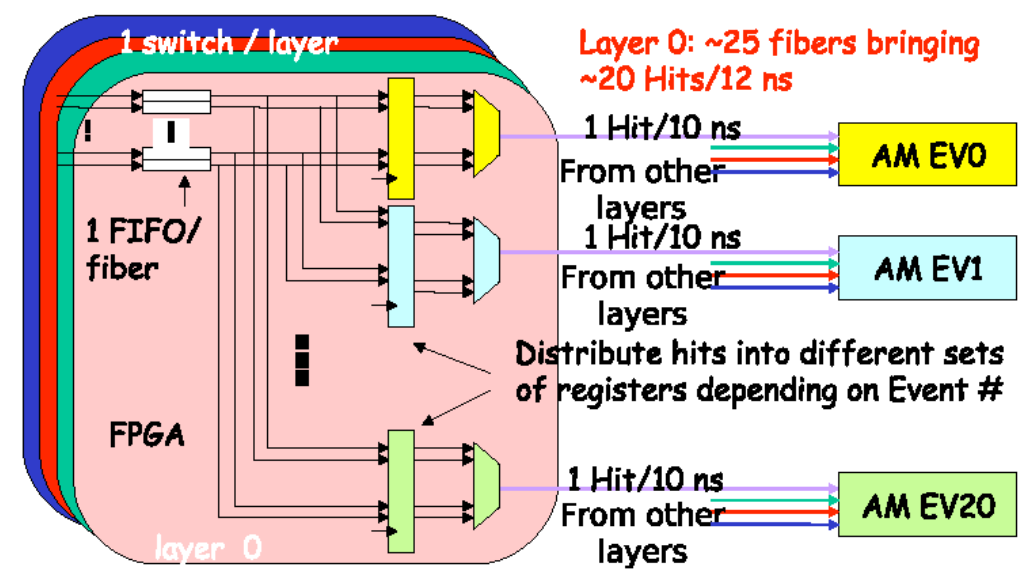

Figure 8. Conceptual design of a possible Tracker Trigger Logic using a switch that distributes hits coming from four layers in a Trigger sector to 20 Associative Memories, one for each event.

\subsection{Dimensioning a possible trigger layout}

In order to prove the effectiveness of such approach a simple exercise has been carried out using a possible layout for the outer part of the Tracker barrel. Five layers of silicon (strip or pixel) sensors are equipped to discriminate against low $\mathrm{p}_{\mathrm{T}}$ tracks with a 90\% rejection; their outputs are binary. The active elements lengths in $\mathrm{z}$ have been taken small enough to reduce the chance of two particles crossing the same set of strips. Strip lengths of about $1 \mathrm{~cm}$ at $50 \mathrm{~cm}$ radius and about $5 \mathrm{~cm}$ for outer layers would guarantee that the strip occupancy is lower than $1 \%$. A majority of 4 out of 5 planes will guarantee a low fake rate and sufficient redundancy against possible missing hits in the track reconstruction.

Hundred trigger sectors are then defined in azimuth, each having the full length in $\mathrm{z}$ to cover a pseudorapidity region of about \pm 1.5 units. This segmentation matches the bending of tracks with $\mathrm{p}_{\mathrm{T}}>10 \mathrm{GeV} / \mathrm{c}$ within a sector. In order to allow a track to be reconstructed in more than one trigger sector, the trigger processors in a slice will receive hits from the two adjacent trigger sectors on each side of it. Such 3-sector structure is referred as super-sector in the following.

Modules in each sector send the reduced information via copper lines to a ASIC located inside the detector, that in turn routes the information outside the detector to the switch via optical fibers. All the hits are sent to the switch together with a unique time stamp to the dedicated AM processor. Each hit is identified with 18 bits word. 
As shown in Table 1, the average data rate per trigger sector is about 2 Gbps. Using a laser driver with 2.56 Gbps speed, working at $80 \%$ payload and allowing for a factor 2.5 safety margin to allow for fluctuations, 2 fibers would accommodate the needs, giving a total number of about 1000 fibers for trigger purposes for the whole Tracker Barrel, to be compared with about 20,000 of the readout of the Tracker Barrel of CMS. Once the Level-1 trigger decision has been taken, the full sensor information needs to be transferred for High Level Trigger purposes, at a $100 \mathrm{KHz}$ rate.

Table 1. Layout of possible Tracker trigger layers in the barrel. Hundred phi trigger sectors are defined, each covering the full barrel length. The average number of single strip clusters is shown together with the required data rate per sector.

\begin{tabular}{|c|c|c|c|c|}
\hline $\begin{array}{c}\text { Radius } \\
(\mathrm{cm})\end{array}$ & $\begin{array}{c}\text { Pitch } \\
(\mu \mathrm{m})\end{array}$ & $\begin{array}{c}\text { Barrel } \\
\text { length } \\
(\mathrm{cm})\end{array}$ & $\begin{array}{c}\text { Average number of } \\
\text { one-strip clusters per } \\
\text { trigger sector/bx }\end{array}$ & $\begin{array}{c}\text { Average data rate per } \\
\text { trigger sector } \\
(\mathrm{Gbps})\end{array}$ \\
\hline 50 & 60 & 140 & 4.0 & 1.4 \\
\hline 65 & 60 & 230 & 5.8 & 2.1 \\
\hline 80 & 80 & 230 & 5.2 & 1.9 \\
\hline 95 & 80 & 230 & 4.8 & 1.7 \\
\hline 108 & 80 & 230 & 4.5 & 1.6 \\
\hline
\end{tabular}

The data rate that needs to be transferred to the AM processor per each sector is therefore the one from Table 1 plus the time stamp (one per sector), made of 12 bits every $50 \mathrm{~ns}$, i.e. $240 \mathrm{Mbps}$. The average number of AM processors that are need to run in parallel (assuming $100 \mathrm{MHz}$ and 18 bits encoding) is about 6 per trigger super-sector. Further and detailed simulations are needed to establish the proper dimensioning of a working system. However the results shown in this Section are encouraging and sets the scale of the problem.

Including Muon or Calorimeter triggers is quite natural with the AM approach. For instance, one of the inputs of the AM chip could be a muon/e- $\gamma$ candidate within the same trigger sector of the Tracker.

Finally it should be noticed that jets done with Level-1 Calorimeters and tracks have already been shown to be very precise [14]. As stressed in Section 3.1 the precise localization of the primary vertex in such jets could help to disentangle those coming from a genuine hard interaction with respect to those coming from minimum bias.

\section{Conclusions}

Tracking information helps reducing drastically the rate of uninteresting events. The CDF SVT experience has become a cornerstone for Tracker Trigger. The CMS upgrade for SLHC will use tracking information for Level-1 trigger purposes. The challenge is enormous due to the high data rate expected. Several approaches are under study, and will provide excellent momentum resolution for tracks above $\sim 10 \mathrm{GeV} / \mathrm{c} \mathrm{p}_{\mathrm{T}}$, muon and electron matching as well as jet reconstruction. A key role will be played by the data reduction at module level, before the information is sent to trigger processors. A model, based on local cluster width discrimination is very attractive; however more $\mathrm{R} \& \mathrm{D}$ is needed to bring it to maturity. The trigger processors will be located offdetector, where the Associative Memories approach (a la CDF) seems feasible. Trigger 
design and detector layout are to some extent connected. Experience from the running of the CMS Tracker and Trigger will give enormous feedback on the ideas for SLHC Trigger.

\section{Acknowledgments}

We would like to thank the CDF experiment for material support and discussions. We thank Paola Giannetti that provided the main ideas for the associative memory architecture at Level-1. We would like to thank the CMS Tracker Colleagues for many interesting discussions and ideas that are presented in this talk.

Finally the speaker wishes to really thank the Organizing Committee for the fantastic atmosphere and charming environment that has allowed a fruitful Conference to happen.

\section{References}

[1] A. Bardi et al., Nucl.Instrum.Meth. A485,178 (2002).

[2] J. Adelman et al., Nucl.Instrum.Meth. A572, 361 (2007).

[3] F. Gianotti et al. Eur. Phys. J. C 39, 293-333 (2005)

[4] M. DellOrso and L. Ristori, Nucl. Intsr. Meth., vol. A278, no. 2, pp. 436-440, Jun. 1989.

[5] R. Amendolia et al., IEEE Trans. on Nucl. Sci., Vol.39, 1992 pp. 795-797.

[6] A. Bardi et al. , Nucl. Intsr. and Meth., vol. A413/2-3, 1998 pp.367-373.

[7] A. Annovi et al., Nuclear Science, IEEE Transactions on Volume 53, 2006, 2428.

[8] R. Field [CDF Collaboration], "Min-bias and the underlying event in Run 2 at CDF," Acta Phys. Polon. B 36 (2005) 167.

[9] F. Palla JINST 2: P02002, 2007

[10] P. Moreira, "The GBT Bi-directional links and Data system", $12^{\text {th }}$ Workshop on Electronics For LHC and Future Experiments,Valencia, Spain, 25 - 29 Sep 2006, pp.93-96

[11] A. Marchioro, private communication

[12] G. Barbagli et al., "Track momentum discrimination using cluster width in silicon strip sensors for SLHC", TWEPP 2007 Topical workshop on electronics for particle physics, Prague, 3-7 September 2007

[13] M. Mannelli, these proceedings

[14] W. Adam et al., Eur. Phys. J. C46 (2006) 605. 